\title{
Esculetin as bioactive marker: towards a rational scientific approach for the treatment of hyperuricemia using Traditional Chinese Medicine
}

\author{
Jieyu Zuo, Wugang Zhang², Hui Jian², Nádia Bou-Chacra ${ }^{3 *}$, Raimar Löbenberg ${ }^{\circledR *}$ \\ ${ }^{1}$ Faculty of Pharmacy and Pharmaceutical Sciences, University of Alberta, Edmonton, AB, Canada. T6G 2E1, ${ }^{2} J i a n g x i$ \\ University of Traditional Chinese Medicine. Nanchang, People's Republic of China, ${ }^{3}$ Faculty of Pharmaceutical Scicences, \\ University of São Paulo, São Paulo, SP, Brazil
}

\begin{abstract}
Traditional Chinese Herbal Medicine has been used to prevent and cure disease in China for thousands of years and has gained global interest in recent decades. The Erding formula is a Chinese Pharmacopeia (ChP)-listed herbal preparation used for treating sore throat, carbuncles and boils. Esculetin is a $\mathrm{ChP}$ quality control (QC) marker for these indications. A previous study found that a new indication, hyperuricemia, can be added to the Erding formula. Therefore, this study aimed to evaluate whether the traditionally used marker, esculetin, still has bioactivity for hyperuricemia, which is substantially different from the original indications. The study analyzed the quantity of esculetin by high-performance liquid chromatography, assessed the therapeutic effect of esculetin using animal model, and then characterized esculetin and its metabolites in serum via ultra-performance liquid chromatography-quadrupole timeof-flight mass spectrometry. The results showed that the esculetin content in the aqueous Erding extract was $0.26 \pm 0.05 \%(w / w)$. Both the Erding extract and esculetin significantly reduced uric acid levels. Six metabolites of esculetin were identified in mice serum. This study revealed a rational scientific approach to prove esculetin is a reliable bioactive and QC marker for Erding formula in hyperuricemia treatment which contributed to ensure product quality and therapeutic efficacy.
\end{abstract}

Keywords: Esculetin. Erding granules. Traditional Chinese Herbal Medicine. Bioactive marker.

\section{INTRODUCTION}

Traditional Chinese Herbal Medicine (TCHM) has been used in China for centuries to prevent and cure disease (Liang et al., 2009). TCHM generally uses several medicinal herbs together, which is quite different from the monotherapies used in Western medicine (Liang et al., 2009; Song et al., 2015). As herbal medicines are gaining popularity worldwide, medicines of consistent and controllable quality are required (Jiang et al., 2010). However, because the composition of herbal products is complex, defining appropriate markers for herbal products remains a challenge for scientists, regulators, and manufacturers (Waldmann et al., 2012). Markers are chemically defined

\footnotetext{
*Correspondence: N. Bou-Chacra. Faculdade de Ciências Farmacêuticas, Universidade de São Paulo, 05508-000 - São Pulo, SP, Brazil. Email: chacra@usp.br

R. Löbenberg. Faculty of Pharmacy and Pharmaceutical Sciences, University of Alberta, Edmonton, AB, T6G 2E1 - Canada.Email: raimar@ualberta.ca
}

constituents of herbal products used for quality control (QC) purposes. Accordingly, there are two categories of markers; active markers, which are accepted to contribute to the therapeutic effect; and analytical markers, which only serve analytical purposes (EMA, 2009). The use of bioactive markers in an herbal product is recommended for QC purposes (Xie et al., 2013). Therefore, for orally administered TCHMs, the components that are absorbed into the systemic circulation and contribute to the therapeutic effect should be considered QC markers (Wang et al., 2011).

Erding granules are an herbal formula containing Viola yedoensis Makino (Viola), Taraxacum mongolicum Hand.-Mazz. (Taraxacum), Lobelia chinensis Lour. (Lobelia), and root of Isatis indigotica Fort. (Isatidis) in equal quantities. According to the Chinese Pharmacopeia (ChP), the Erding formula is used to treat sore throat, carbuncles and boils due to its heat-clearing and detoxifying properties. Esculetin is a QC marker for the original Erding granule indications listed above, based 
on content (NPC, 2015). This substance is a coumarin that exhibits antibacterial, anti-inflammatory, sedative, anticonvulsive, analgesic, antitussive, expectorant, and antiasthmatic effects (Zhu, 1998). Hence, for the original indication of the Erding formula, esculetin could be regarded as an active marker. Previous studies found that this formula has the potential for treating hyperuricemia (Wang et al., 2016, Zuo et al., 2018). Hyperuricemia is an abnormally high concentration of uric acid (UA) in the body caused by a purine metabolic disorder. This disease is a risk factor for gout, hypertension, diabetes mellitus, stroke, kidney failure and cardiovascular events (Liu et al., 2015). Population-based studies have estimated a prevalence of up to $21.0 \%$ for hyperuricemia in Western countries, and the prevalence of hyperuricemia in mainland China is $13.3 \%$ (Liu et al., 2015; Bove et al., 2017). In treating hyperuricemia, esculetin might only be an analytical marker instead of a relevant marker of in vivo activity.

Ultra-high performance liquid chromatographyquadrupole time-of-flight mass spectrometry (UHPLC/ Q-TOF-MS/MS) is a very rapid, sensitive, powerful and reliable analytical method for characterizing the absorbed constituents and metabolites of TCHMs. This method can provide excellent chromatography separation with accurate mass measurements of precursor and metabolite ions to identify the main and/or trace substances in herbal formulas (Cai et al., 2013; Xu et al., 2017).

Therefore, in this study, we aimed to identify whether the pre-existing marker esculetin is a potential bioactive and QC marker for the Erding formula when used to treat hyperuricemia by investigating its therapeutic effects in an animal model and identifying its metabolites in serum.

\section{MATERIAL AND METHODS}

\section{Reagents}

Oxonic acid potassium salt (Lot: BCBN6836V) was purchased from Sigma Aldrich (St. Louis, MO, USA). Allopurinol tablets (Lot: 05150603) were purchased from Shanghai SINE Pharm Co., Ltd. (Shanghai, China). The UA assay kit was obtained from Nanjing Jiancheng Bioengineering Institute (Nanjing, China). The esculetin (Figure 1) (BCTG-0523) standard was obtained from the Jiangxi Herbfine Co., Ltd. (Nanchang, China). Methanol and acetonitrile used in the experiments were of highperformance liquid chromatography (HPLC) grade and were obtained from Fisher Scientific (Pittsburgh, PA, USA). Other reagents were of analytical grade.

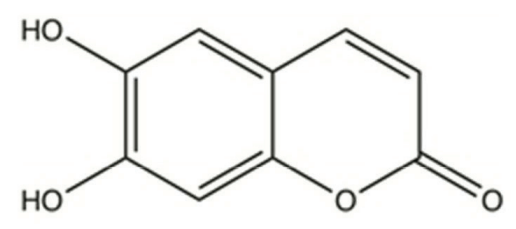

Esculetin

FIGURE 1 - Chemical structure of esculetin.

\section{Plant materials}

The commercial available dry components of Erding granules including Viola (lot: 150309), Taraxacum (lot: 150420), Lobelia (lot: 150417), and Isatidis (lot: 150317) were obtained from the Jiangxi Provincial Hospital of Traditional Chinese Medicine, Nanchang, China. The voucher specimens of the four herb materials were deposited at the Herbarium of the Jiangxi University of Traditional Chinese Medicine with the number 150705.

\section{Preparation of the Erding aqueous extract}

The Erding extract composition was based on the recipe in the $\mathrm{ChP}$ (NPC, 2015). All four herbs were mixed in equal quantities. The mixtures were decocted with distilled water $(1: 10, \mathrm{w} / \mathrm{v})$ under reflux for $2 \mathrm{~h}$, and the supernatant was filtered. The extraction process was immediately repeated with the residue material for another $1.5 \mathrm{~h}$ with the same quantity of menstruum and was followed by filtering. The combined two filtrates were concentrated under vacuum, and the remaining solution was spray-dried. The extract yield was $32.20 \%$ (w/w).

\section{HPLC assessment of esculetin content in the Erding extract}

The HPLC assay was performed with a slight modification in accordance with the $\mathrm{ChP}$ chapter on Erding granules (NPC, 2015). The esculetin content in the Erding extract was determined using a Shimadzu LC-20ADXR HPLC instrument (Kyoto, Japan) with a Welch Ultimate ${ }^{\circledR}$ (Shanghai, China) XB-C18 column $(4.6 \mathrm{~mm} \times 250 \mathrm{~mm}, 5 \mu \mathrm{m})$. The mobile phase consisted of acetic acid: water: methanol $(0.4: 70: 30, \mathrm{v} / \mathrm{v})$. The flow rate was $1.0 \mathrm{~mL} / \mathrm{min}$, the column temperature was $25^{\circ} \mathrm{C}$, and the injection volume was $10 \mu \mathrm{L}$. The UV detector wavelength was set to $353 \mathrm{~nm}$ according to the ChP (NPC, 2015). The run time for each sample was $20 \mathrm{~min}$, and the retention time of esculetin was approximate $10.8 \mathrm{~min}$. The standard esculetin solutions were prepared in methanol at concentrations ranging from 0.0042 to $0.042 \mu \mathrm{g} / \mu \mathrm{L}$. The 
Erding extract was completely dissolved and prepared in water at $9.98 \mu \mathrm{g} / \mu \mathrm{L}$. Then, all the solutions were filtered $(0.45 \mu \mathrm{m})$ and transferred into auto-sampler vials. A $10-\mu \mathrm{L}$ aliquot was injected into the system for analysis. Three replicates were performed on independent samples $(n=3)$. The peak area was used to quantify the esculetin content in the Erding extract. All the data were acquired and processed using Lab Solution software (Shimadzu, Kyoto, Japan).

\section{Animals}

All the studies were performed in accordance with the Regulations of the Experimental Animal Administration issued by the State Committee of Science and Technology of the People's Republic of China and were approved by the University Committee on the Use and Care of Animals, Jiangxi University of Traditional Chinese Medicine. Specific pathogen-free male Kunming mice (body weight 14-16 g) were obtained from the Shanghai Slac Laboratory Animal Co., Ltd. (Shanghai, China). These animals were housed in an air-conditioned room at $22 \pm 2{ }^{\circ} \mathrm{C}$ with a relative humidity of $50 \pm 20 \%$. Standard food and water were provided under a natural light-dark cycle for 3 days to allow the animals to adapt to their environment before the experiment.

\section{Hyperuricemic mouse model}

The hyperuricemic animal model was induced by a uricase inhibitor, potassium oxonate (Hall et al., 1990). The mice were randomly divided into 5 groups $(n=10)$ : the untreated (healthy mice), untreated disease, positive control (allopurinol), Erding extract, and esculetin groups. Potassium oxonate was freshly suspended in $0.5 \%(\mathrm{w} / \mathrm{v})$ sodium carboxymethyl cellulose (CMC-Na) solution before use. Except for the normal control mice, which received a $0.5 \%(\mathrm{w} / \mathrm{v}) \mathrm{CMC}-\mathrm{Na}$ solution, the mice were intraperitoneally injected with potassium oxonate $(450 \mathrm{mg} / \mathrm{kg}) 1 \mathrm{~h}$ before the last dose to enhance the serum UA level.

\section{Treatment}

The extract was dissolved in distilled water, and the administered volume to mice was $20 \mathrm{~mL} / \mathrm{kg}$ body weight. The dose to mice was expressed as gram of extract per kilogram of body weight. In this study, the Erding extract dose was $7.73 \mathrm{~g} / \mathrm{kg}$ body weight. The esculetin dose was equal to its content in the Erding extract. The esculetin was freshly suspended in water before use. The extract and esculetin were administered to the mice for five consecutive days by gavage. Simultaneously, allopurinol was administered orally at $15 \mathrm{mg} / \mathrm{kg}$ body weight as a positive control. The untreated and untreated disease groups were orally administered water for five consecutive days. Food but not water was withdrawn from the mice $1 \mathrm{~h}$ prior to administration except for the last day, on which the food was withdrawn $12 \mathrm{~h}$ before administration.

\section{Blood sample collection and UA assay of hyperuricemic mice}

Blood samples were obtained from eyes and collected in $1.5 \mathrm{~mL}$ Eppendorf tubes $1 \mathrm{~h}$ after the last dose. All blood samples were allowed to clot at room temperature for $1 \mathrm{~h}$ before centrifugation at $1,400 \mathrm{x} \mathrm{g}$ for $10 \mathrm{~min}$ to obtain serum. The serum was collected and stored at $-20{ }^{\circ} \mathrm{C}$ until analysis. After blood sample collection, all the mice were sacrificed by cervical dislocation. The serum UA level was determined using the phosphotungstic acid method following the manufacturer's protocol (Carroll et al., 1971). Except for the untreated and the positive control groups, residual serum (untreated disease, Erding extract, and esculetin groups) were stored at $-80{ }^{\circ} \mathrm{C}$ until metabolite analysis.

\section{Assessing Erding extract metabolites by UPLC/ Q-TOF-MS/MS}

\section{UHPLC/Q-TOF-MS/MS system conditions}

The esculetin metabolites of both Erding extract group and esculetin group were identified using a Shimadzu UHPLC LC-30AD system (Kyoto, Japan) coupled with an AB Sciex Triple $\mathrm{TOF}^{\mathrm{TM}} 5600^{+}$mass spectrometer (Foster City, CA, USA) with a DuoSpray ${ }^{\mathrm{TM}}$ ion source. The serum samples were separated using a Waters ACQUITY ${ }^{\circledR}$ UPLC BEH C 18 column $(2.1 \mathrm{~mm} \times 100 \mathrm{~mm}, 1.7 \mu \mathrm{m}$, Milford, MA, USA) with an acetonitrile gradient at room temperature. The mobile phase consisted of phase A (water containing $0.1 \%$ formic acid) and phase B (acetonitrile), and the elution gradient was set as follows: $5 \%-65 \% \mathrm{~B}$ at $0.1-15.0 \mathrm{~min}$, $65-100 \% \mathrm{~B}$ at $15.1-37.0 \mathrm{~min}$, and $5 \% \mathrm{~B}$ at $37.1-40.0 \mathrm{~min}$. The flow rate was $0.3 \mathrm{~mL} / \mathrm{min}$, and the injected sample size was $5 \mu \mathrm{L}$. The mass spectra were acquired in negative ion mode with the following parameters: ion spray voltage: $-4500 \mathrm{~V}$ (negative ion), nebulizer gas: 50 psi, turbo gas: 50 psi, curtain gas (nitrogen): 25 psi, mass range: $\mathrm{m} / \mathrm{z}$ 50-1250, accumulation time: $300.0 \mathrm{~ms}$. The obtained data were analyzed using PeakView ${ }^{\circledR}$ Software 1.2 (Foster City, CA, USA). 


\section{UHPLC/Q-TOF-MS/MS sample preparation}

The serum samples (100 $\mu \mathrm{L}$ each) from untreated disease, Erding extract, and esculetin groups were mixed with $500 \mu \mathrm{L}$ of methanol in a $1.5-\mathrm{mL}$ Eppendorf tube. The mixture was vortexed for $5 \mathrm{~min}$ and then centrifuged at $16,000 \mathrm{x} g$ for $10 \mathrm{~min}$. The supernatant was transferred to a new 1.5-mL Eppendorf tube and evaporated to dryness under nitrogen. The dry residue was re-dissolved in $100 \mu \mathrm{L}$ of acetonitrile: water $(50: 50, \mathrm{v} / \mathrm{v})$ and vortexed for $5 \mathrm{~min}$. Finally, the resulting solution was filtered $(0.45 \mu \mathrm{m})$ and transferred to an auto-sampler vial. A 5- $\mu \mathrm{L}$ aliquot was injected into the system for analysis. Three independent replicates were performed for each group $(n=3)$.

\section{Statistical analysis}

All data are expressed as the mean \pm standard deviation of the mean from 10 mice per group. Statistical comparisons were performed by a t-test with $\alpha=0.05$ using Minitab 15 (State College, PA, USA).

\section{RESULTS AND DISCUSSION}

\section{HPLC profile of the Erding extract}

The correlation coefficient of the calibration curve $(\mathrm{y}=50054193.1217 \mathrm{x}-6563.9444, \mathrm{n}=6)$ was 0.9999 over the $0.0042-0.042 \mu \mathrm{g} / \mu \mathrm{L}$ concentration range, with a coefficient variation of $0.27 \%$. The HPLC profile of the esculetin in the Erding extract is shown in Figure 2. The esculetin content in the Erding extract was $0.26 \pm 0.05 \%(w / w)$. For the QC of the Erding formula in the ChP, the content of esculetin should be no less than $0.09 \%$ (w/w) (NPC, 2015).
In the present study, the esculetin quantity is sufficient for the QC of the Erding formula. As such, the extract could be used to evaluate therapeutic efficacy in a hyperuricemic animal model.

\section{Effects of esculetin and the Erding extract in mice with potassium oxonate-induced hyperuricemic mice}

The oxonate-induced hyperuricemic mouse model was used to investigate the hypouricemic effect of esculetin and Erding extract. This model has been used for decades to evaluate potentially medicinal substances that influence UA levels (Osada et al., 1993; Mo et al., 2007). Throughout evolution, humans have lost uricase, an enzyme that breaks down UA to allantoin, while other mammals, such as rodents, have not. To mimic this human condition in a rodent model, a uricase inhibitor, such as potassium oxonate, which blocks the conversion of UA to allantoin, was chosen to accumulate and increase UA levels in rodents (Kou et al., 2016). In this study, we used a colorimetric method in which a phosphotungstic reagent oxidized the UA to allantoin (Carroll et al., 1971) to determine the UA levels. This method was used because other existing methods for measuring UA may be impacted by potassium oxonate, as it is used to determine the concentration of hydrogen peroxide formed by oxidizing UA to allantoin under the catalysis of uricase (Domagk, Schlicke, 1968; Gochman, Schmitz, 1971).

According to the dose of Erding $(7.73 \mathrm{~g} / \mathrm{kg}$ body weight) and the content of esculetin in the Erding extract $(0.26 \pm 0.05 \% \mathrm{w} / \mathrm{w})$, the esculetin dose administered to mice was $20 \mathrm{mg} / \mathrm{kg}$ body weight. The effects of esculetin

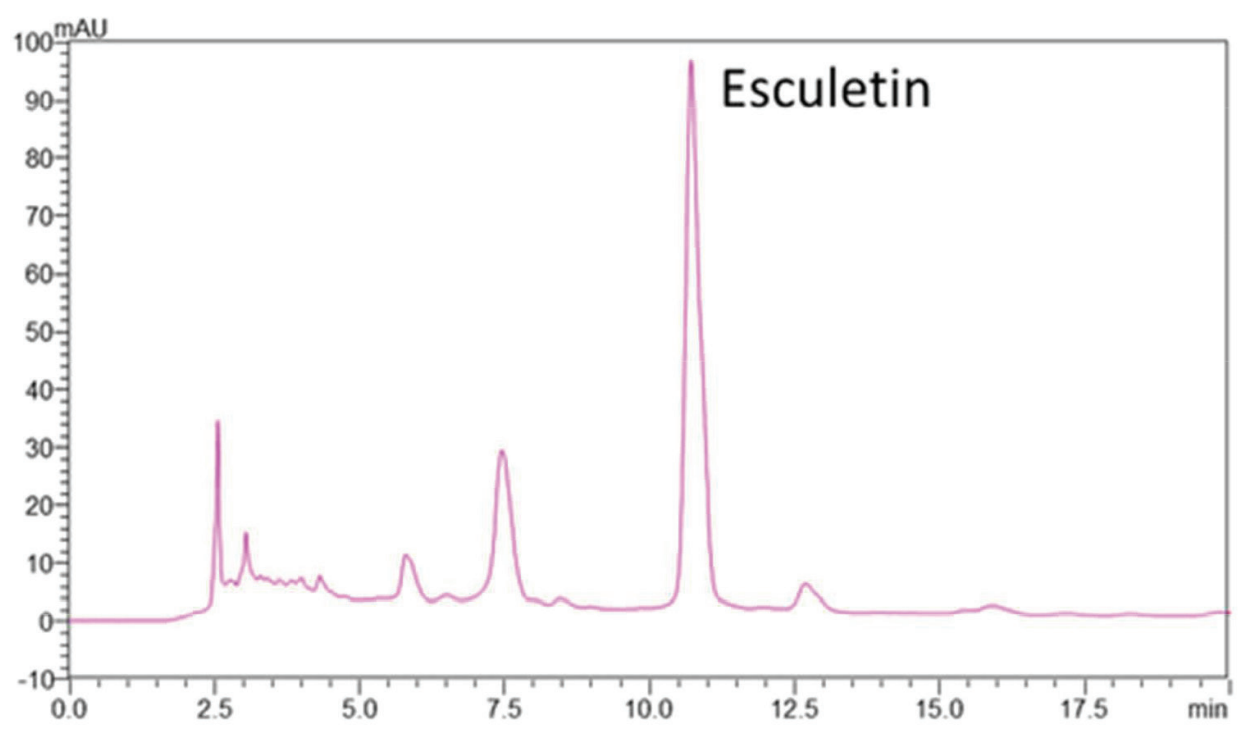

FIGURE 2 - Chromatogram of the esculetin (retention time: $10.8 \mathrm{~min}$ ) in the Erding extract at the detected wavelength, $353 \mathrm{~nm}$. 
on the UA levels are shown in Figure 3. Examination of the results demonstrates that intraperitoneally injected potassium oxonate significantly elevated the serum UA levels ( $p$-value $<0.01, \alpha=0.05$ ). However, allopurinol and the Erding extract significantly decreased the UA levels in hyperuricemic mice ( $\mathrm{p}$-value $<0.01, \alpha=0.05$ ). The orally administered esculetin suspension also reduced the UA levels compared with the untreated disease group ( $p$-value $<0.05, \alpha=0.05$ ). The results showed the treatment with either the Erding formula or esculetin was determined to exert hypouricemic effects by measuring the UA levels. The effect of Erding formula on reducing UA levels has been shown in previous study (Zuo et al., 2018). Esculetin can be one of the active compounds when Erding formula was used to treat hyperuricemia.

\section{Identification of esculetin and its metabolites in serum using UPLC/Q-TOF-MS/MS}

UHPLC/Q-TOF-MS/MS was employed to identify the metabolites of esculetin in dosed serum of mice. It is a very reliable analytical method for identifying trace amounts of absorbed constituents and metabolites of TCHM formulas. The method can provide excellent chromatography separation with accurate mass measurements of precursor and metabolite ions to identify the main and/or trace substances of herbal formulas. The electrospray ionization-MS and collisioninduced dissociation-MS/MS conditions were optimized to obtain suitable fragment ions for the identification of esculetin and its metabolites in serum. Welch UHPLC C18 $(100 \mathrm{~mm} \times 2.1 \mathrm{~mm}, 1.8 \mu \mathrm{m})$ and Waters ACQUITY UPLC BEH C18 (100 mm x $2.1 \mathrm{~mm}, 1.7 \mu \mathrm{m})$ columns were investigated to obtain a better resolution and peak shape from the substances. Finally, the negative ion mode and
Waters ACQUITY UPLC BEH C18 column were selected according to the higher MS response of esculetin and its metabolites and the better separation efficacy. Formic acid was added to the mobile phase to inhibit insufficient substance ionization.

The full-scan mass spectrum of fragment ions in mouse serum after administration of the Erding extract or esculetin was compared with the spectra obtained from untreated disease serum samples to identify esculetin and its possible metabolites. As shown in Figure 4, the peaks detectable in the serum of Erding extract- and esculetintreated hyperuricemic mice, but not in the serum of mice in the untreated disease control group, could be as attributed to esculetin and its metabolites. Using the PeakView 1.2 software EIC function, we obtained the relative peak areas (Figure 4) of esculetin and possible metabolites (M1-M6).

The spectra of esculetin and its metabolites (M1-M6) exhibited [M-H] ${ }^{-}$at $\mathrm{m} / \mathrm{z} 177.019,353.051,256.976$, 270.992, 367.067, 529.084, and 433.008, respectively, which indicated the formulas were $\mathrm{C}_{9} \mathrm{H}_{6} \mathrm{O}_{4}$ (esculetin), $\mathrm{C}_{15} \mathrm{H}_{14} \mathrm{O}_{10}$ (M1), $\mathrm{C}_{9} \mathrm{H}_{6} \mathrm{O}_{7} \mathrm{~S}$ (M2), $\mathrm{C}_{16} \mathrm{H}_{16} \mathrm{O}_{10}$ (M3), $\mathrm{C}_{21} \mathrm{H}_{22} \mathrm{O}_{16}$ (M4), $\mathrm{C}_{21} \mathrm{H}_{22} \mathrm{O}_{16}$ (M5) and $\mathrm{C}_{15} \mathrm{H}_{14} \mathrm{O}_{13} \mathrm{~S}$ (M6), as shown in Table I and Figure 5.

When a substance is absorbed into the circulation, enzymes cause further metabolization. Generally, the metabolism can be divided into two phases. Phase I reactions mainly include modification via oxidation, reduction, hydrolysis, cyclization, and decyclization, as well as oxygen addition or hydrogen removal. Phase II reactions mainly occur at carboxyl, hydroxyl, amino, and sulfhydryl groups via conjugation with such molecules as glutathione, sulfate, glycine, and glucuronic acid. (Xu et al., 2017)

Esculetin is the simplest coumarin, with two hydroxyl groups at carbons 6 and 7 (as shown in Figure 1),

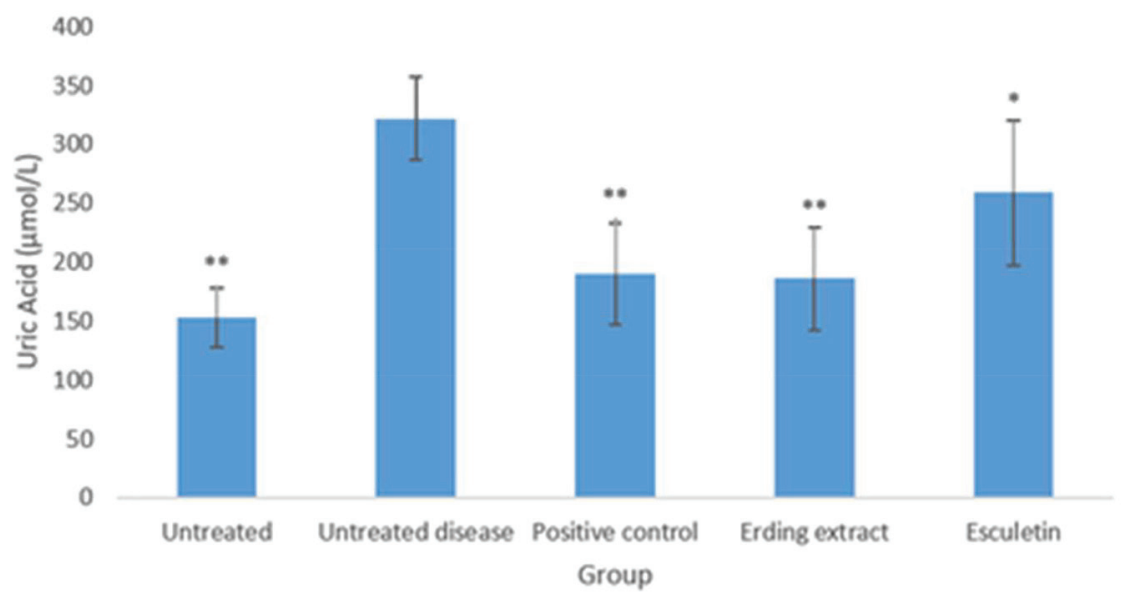

FIGURE 3 - Effects of esculetin and the Erding extract on the serum UA levels in mice with potassium oxonate-induced hyperuricemia. ${ }^{*}$ p-value $<0.05$ and $* * p$-value $<0.01$ compared with the untreated disease group. 
which suggests that the metabolism of esculetin in vivo is probably mainly a phase II reaction because the hydroxyl groups may be easily substituted. In the MS/MS spectra (Figure 5), M1, M4, and M6 lost $176 \mathrm{Da}$ to produce fragments at $\mathrm{m} / \mathrm{z} 177,191$, and 257 , respectively. M5 could lose $176 \mathrm{Da}$ twice to produce fragments at $\mathrm{m} / \mathrm{z} 353$ and 177. These results indicate that one or two glucuronic acids were dissociated with metabolites. For M2, M3, and M6, the loss of SO3 (80 Da) suggests sulfate group conjugation. The loss of $14 \mathrm{Da}(\mathrm{CH} 2)$ in M3 and M4 suggests the occurrence of methylation. Therefore, each or both of the hydroxyl groups of esculetin were modified by glycosylation, sulfation and/or methylation after drug administration and absorption from the gastrointestinal tract. The identification and characterization of M1M5, as shown in Figure 5, is consistent with previously reported data from Ding et al. (2009). We hypothesized that the glucuronide (M1) and sulfation (M2) metabolites

TABLE I - Accurate mass measurement of protonated metabolites in mouse serum samples in negative ion mode

\begin{tabular}{lcccccc}
\hline No. & Formula & $\mathbf{T}_{\mathbf{R}}(\mathbf{m i n})$ & Ion mode & Measured mass & Error (ppm) & Metabolite description \\
\hline $\mathrm{P}$ & $\mathrm{C}_{9} \mathrm{H}_{6} \mathrm{O}_{4}$ & 3.52 & {$[\mathrm{M}-\mathrm{H}]^{-}$} & 177.019 & -1.4 & Parent esculetin \\
M1 & $\mathrm{C}_{15} \mathrm{H}_{14} \mathrm{O}_{10}$ & 3.10 & {$[\mathrm{M}-\mathrm{H}]^{-}$} & 353.051 & -0.4 & Glucuronide \\
$\mathrm{M} 2$ & $\mathrm{C}_{9} \mathrm{H}_{6} \mathrm{O}_{7} \mathrm{~S}$ & 3.52 & {$[\mathrm{M}-\mathrm{H}]^{-}$} & 256.976 & -1.0 & Sulfation \\
M3 & $\mathrm{C}_{10} \mathrm{H}_{8} \mathrm{O}_{7} \mathrm{~S}$ & 4.01 & {$[\mathrm{M}-\mathrm{H}]^{-}$} & 270.992 & -3.2 & Sulfation and methylation \\
M4 & $\mathrm{C}_{16} \mathrm{H}_{16} \mathrm{O}_{10}$ & 3.72 & {$[\mathrm{M}-\mathrm{H}]^{-}$} & 367.067 & -0.9 & Glucuronide and methylation \\
M5 & $\mathrm{C}_{21} \mathrm{H}_{22} \mathrm{O}_{16}$ & 2.07 & {$[\mathrm{M}-\mathrm{H}]^{-}$} & 529.084 & 0.8 & Diglucuronide \\
M6 & $\mathrm{C}_{15} \mathrm{H}_{14} \mathrm{O}_{13} \mathrm{~S}$ & 2.54 & {$[\mathrm{M}-\mathrm{H}]^{-}$} & 433.008 & -0.6 & Glucuronide and sulfation \\
\hline
\end{tabular}
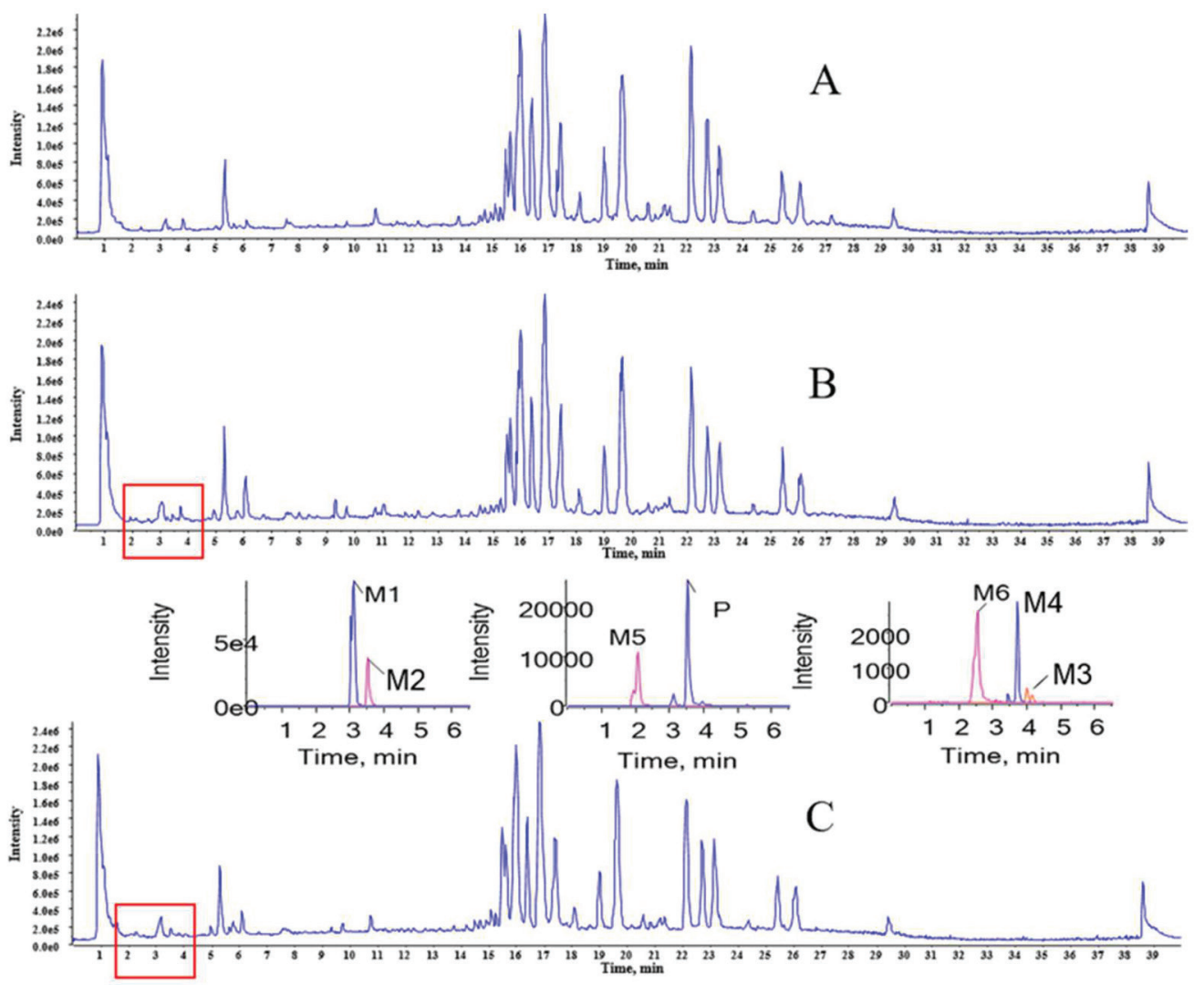

FIGURE 4 - Total ion chromatogram and extract ion chromatogram of esculetin and metabolites from mice serum samples in negative ion mode. (A) Untreated hyperuricemic mice; (B) Erding extract-treated hyperuricemic mice; (C) esculetin-treated hyperuricemic mice. P: parent esculetin $\mathrm{C}_{9} \mathrm{H}_{6} \mathrm{O}_{4}$; M1: glucuronide metabolite $\mathrm{C}_{15} \mathrm{H}_{14} \mathrm{O}_{10}, \mathrm{M} 2$ : sulfation metabolite $\mathrm{C}_{9} \mathrm{H}_{6} \mathrm{O}_{7} \mathrm{~S}$; $\mathrm{M} 3$ : sulfation and methylation metabolite $\mathrm{C}_{10} \mathrm{H}_{8} \mathrm{O}_{7} \mathrm{~S} ;$ M4: glucuronide and methylation metabolite $\mathrm{C}_{16} \mathrm{H}_{16} \mathrm{O}_{10}$; M5: diglucuronide metabolite $\mathrm{C}_{21} \mathrm{H}_{22} \mathrm{O}_{16}$; M6: glucuronide and sulfation metabolite $\mathrm{C}_{15} \mathrm{H}_{14} \mathrm{O}_{13} \mathrm{~S}$. 


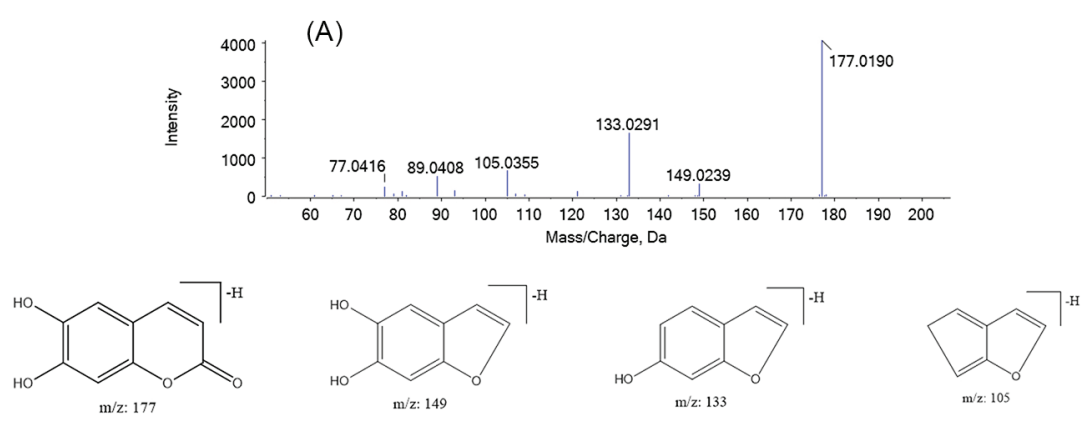

M1 (B)

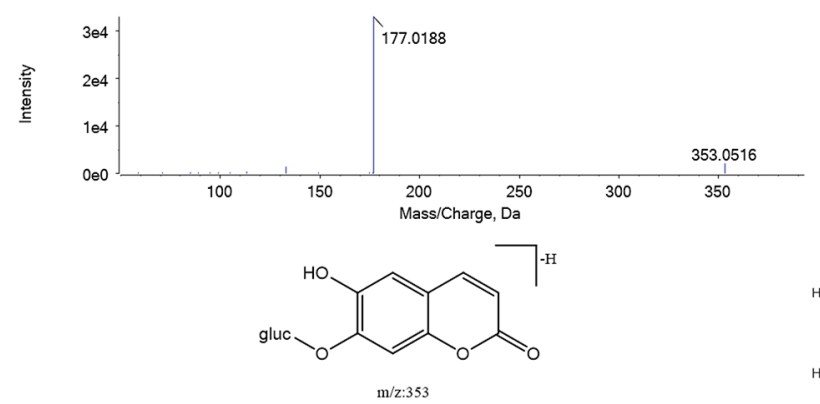

м3 (D)
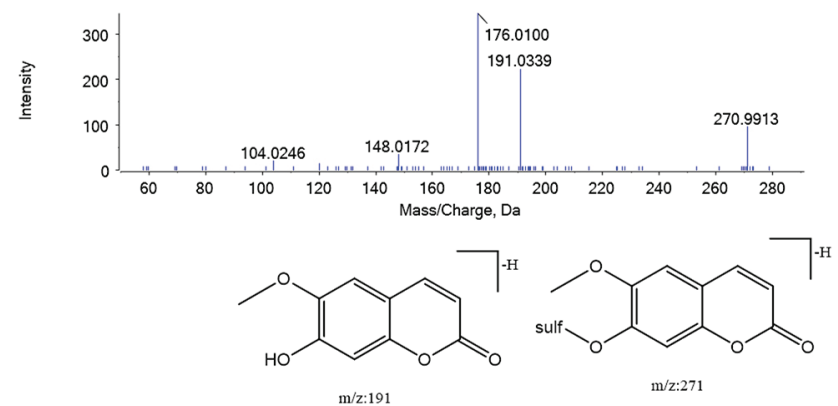

M5 $(F)$

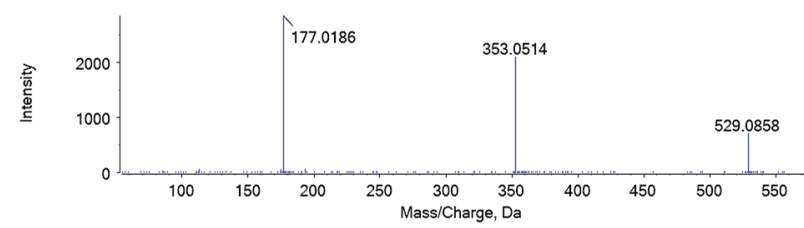

(-H)

m/2: 177<smiles>CCc1cc2cc(O)c(OC)cc2oc1=O</smiles>

$\mathrm{m} / \mathrm{z}: 353$<smiles>CCCCCCC</smiles>

m/2::29
M2 (C)

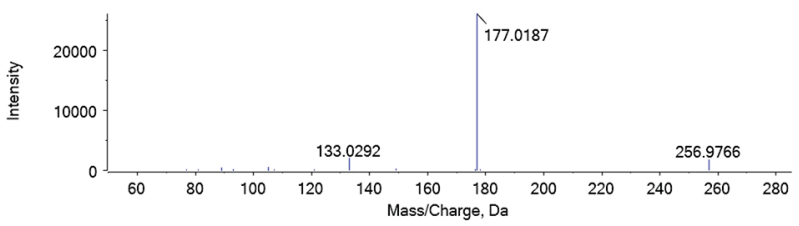

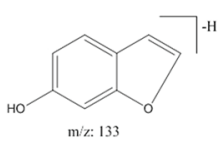

m/z: 177

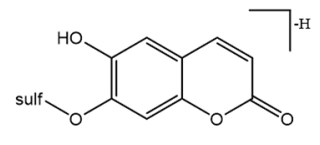

$\mathrm{m} / \mathrm{z}: 257$

M4 (E)

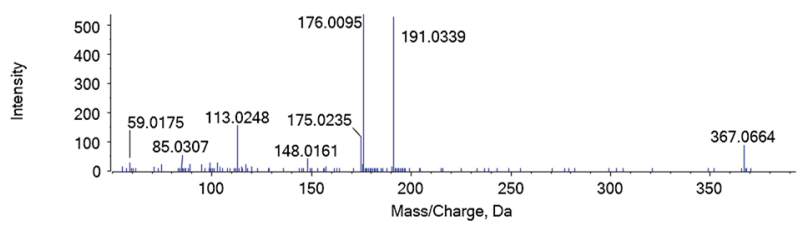<smiles>CC[CH]CC</smiles>

M6

(G)

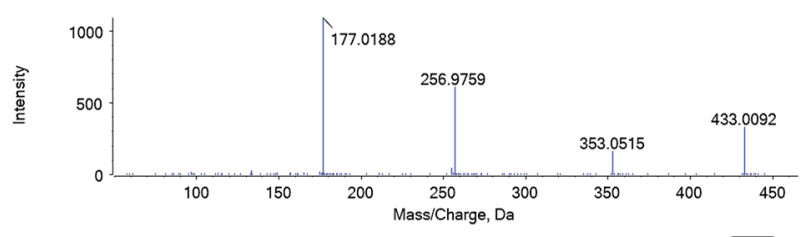

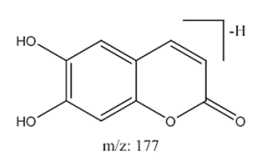

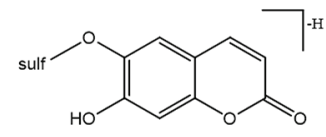

$\mathrm{m} / 2: 257$<smiles>CCOc1cc2oc(=O)ccc2cc1O</smiles>

m/z:353<smiles>CCCCCCC</smiles>

FIGURE 5 - Mass spectra (MS/MS) of esculetin and its possible metabolites. (A) Esculetin, (B) M1, (C) M2, (D) M3, (E) M4, (F) M5, and (G) M6.

of esculetin observed in serum may be the major metabolites in the circulation, depending on the intensity of the responses, as shown in Figure 4. These findings demonstrate that esculetin is one of the compounds within the Erding extract that is absorbed into the systemic circulation.

As the use of herbs and natural-origin products becomes more common not only in Asia but also in Western 
countries, proper product identification and QC is becoming increasingly necessary (Xie, Leung, 2009). For TCHMs, an ideal QC marker should not only be a high-content analytical marker but also a constituent responsible for the biological and/or therapeutic effects of the treatment (Jiang et al., 2009). When a new therapeutic use for a formula is added, whether the original marker is still a suitable bioactive marker or a new marker is required should be investigated. For the treatment of hyperuricemia, flavonoids are a group of phytochemicals that are potent alternatives to allopurinol (Cos et al., 1998; Nagao, Seki, Kobayashi, 1999; Zhu et al., 2004). However, for the Erding formula, the content of flavonoids and/or a specific representative flavonoid are not clear. Additionally, according to the $\mathrm{ChP}$ and a previous study, esculetin is a pre-existing and relatively high-content QC marker for the Erding formula (NPC,2015; Zhan et al., 2015). In this case, esculetin is an appropriate bioactive and QC marker for the Erding extract in treating hyperuricemia.

Most herbal products are orally administered; however, phytoequivalence is somewhat theoretical when herbs and their products are developed or compared with other products. In addition to the lack of established reference products, some specific issues should also be considered. Unlike conventional/orthodox Western medicines containing one or a few combinations of defined active pharmaceutical ingredients, herbal formulas are more complex, and most of the ingredients are unknown. Herbal extracts can contain constituents solely responsible for the therapeutic activity, chemically defined constituents possessing active markers, or chemical markers only. Hence, it is valuable to confirm that the most appropriate biomarker is chosen to ensure the quality of herbal products upon the addition of a new indication (Waldmann et al., 2012).

\section{CONCLUSION}

In this study, a rational scientific approach based on UHPLC/Q-TOF-MS/MS revealed that esculetin is an appropriate bioactive QC marker for Erding formula in hyperuricemia treatment. These findings will not only contribute to improve the modern TCHM preparations quality but also will help to ensure therapeutic efficacy for patients.

\section{ACKNOWLEDGMENT}

The authors thank the International Cooperative Scientific Grant of Jiangxi Province, China (No. 20144BDH80005 and 20151BBB70268) for financial support.

\section{REFERENCES}

Bove M, Cicero AF, Veronesi M, Borghi C. An evidence-based review on urate-lowering treatments: Implications for optimal treatment of chronic hyperuricemia. Vasc Health Risk Manag. 2017;13:23-8.

Cai Y, Gao Y, Tan G, Wu S, Dong X, Lou Z, et al. Myocardial lipidomics profiling delineate the toxicity of traditional Chinese medicine Aconiti Lateralis radix praeparata. J Ethnopharmacol. 2013;147(2):349-56.

Carroll JJ, Coburn H, Douglass R, Babson AL. A simplified alkaline phosphotungstate assay for uric acid in serum. Clin Chem. 1971;17(3):158-60.

Cos P, Ying L, Calomme M, Hu JP, Cimanga K, Van Poel $\mathrm{B}$, et al. Structure-activity relationship and classification of flavonoids as inhibitors of xanthine oxidase and superoxide scavengers. J Nat Prod. 1998;61(1):71-6.

Ding WJ, Deng Y, Feng H, Liu WW, Hu R, Li X, et al. Biotransformation of aesculin by human gut bacteria and identification of its metabolites in rat urine. World $\mathrm{J}$ Gastroenterol. 2009;15(12):1518-23.

Domagk GF, Schlicke HH. A colorimetric method using uricase and peroxidase for the determination of uric acid. Anal Biochem. 1968;22(2):219-24.

European Medicine Agency, 2009. Guideline on quality of combination herbal medicinal products / traditional herbal medicinal products. [Accessed on Nov 25, 2016.] Available from: http://www.ema.europa.eu/docs/en_GB/document_ library/Scientific_guideline/2009/09/WC500003286.pdf.

Gochman N, Schmitz JM. Automated determination of uric acid, with use of a uricase-peroxidase system. Clin Chem. 1971;17(12):1154-9.

Hall IH, Scoville JP, Reynolds DJ, Simlot R, Duncan P. Substituted cyclic imides as potential anti-gout agents. Life Sci. 1990;46(26):1923-7.

Jiang Y, David B, Tu P, Barbin Y. Recent analytical approaches in quality control of traditional Chinese medicines--A review. Anal Chim Acta. 2010;657(1):9-18.

Kou Y, Li Y, Ma H, Li W, Li R, Dang Z. Uric acid lowering effect of Tibetan Medicine RuPeng15 powder in animal models of hyperuricemia. J Tradit Chin Med. 2016;36(2):205-10. 
Liang XM, Jin Y, Wang YP, Jin GW, Fu Q, Xiao YS. Qualitative and quantitative analysis in quality control of traditional Chinese medicines. J Chromatogr A. 2009;1216(11):2033-44.

Liu R, Han C, Wu D, Xia X, Gu J, Guan H, et al. Prevalence of hyperuricemia and gout in Mainland China from 2000 to 2014: A systematic review and meta-analysis. BioMed Res Int. 2015;2015:762820.

Mo SF, Zhou F, Lv YZ, Hu QH, Zhang DM, Kong LD. Hypouricemic action of selected flavonoids in mice: Structureactivity relationships. Biol Pharm Bull. 2007;30(8):1551-6.

Nagao A, Seki M, Kobayashi H. Inhibition of xanthine oxidase by flavonoids. Biosci Biotechnol Biochem. 1999;63(10):1787-90.

National Pharmacopoeia Committee. Erding Granules. In: Pharmacopoeia of the People's Republic of China, vol. 1. Beijing: Chemical Industry Press; 2015. p.435.

Osada Y, Tsuchimoto M, Fukushima H, Takahashi K, Kondo S, Hasegawa M, et al. Hypouricemic effect of the novel xanthine oxidase inhibitor, TEI-6720, in rodents. Eur J Pharmacol. 1993;241(2-3):183-8.

Song Y, Zhang N, Shi S, Li J, Zhang Q, Zhao Y, et al. Largescale qualitative and quantitative characterization of components in Shenfu injection by integrating hydrophilic interaction chromatography, reversed phase liquid chromatography, and tandem mass spectrometry. J Chromatogr A. 2015;1407:106-18.

Waldmann S, Almukainzi M, Bou-Chacra N, Amidon GL, Lee BJ, Feng J, et al. Provisional biopharmaceutical classification of some common herbs used in western medicine. Mol Pharm. 2012;9(4):815-22.

Wang L, Wang Z, Wo S, Lau CB, Chen X, Huang M, et al. A bio-activity guided in vitro pharmacokinetic method to improve the quality control of Chinese medicines, application to $\mathrm{Si} \mathrm{Wu}$ Tang. Int J Pharm. 2011;406(1-2):99-105.
Wang M, Xu X, Wu C, Gong Q, Zhang W, Jian H, et al. Pharmacodynamics study of Erding granule for gout. Pharmacol Clin Chin Materia Med. 2016;32:125-8. (Article in Chinese).

Xie C, Wang Z, Wang C, Xu J, Wen Z, Wang H, et al. Utilization of gene expression signature for quality control of traditional Chinese medicine formula Si-Wu-Tang. AAPS J. 2013;15(3):884-92.

Xie PS, Leung AY. Understanding the traditional aspect of Chinese medicine in order to achieve meaningful quality control of Chinese materia medica. J Chromatogr A. 2009;1216(11):1933-40.

Xu T, Li S, Sun Y, Pi Z, Liu S, Song F, et al. Systematically characterize the absorbed effective substances of Wutou Decoction and their metabolic pathways in rat plasma using UHPLC-QTOF-MS combined with a target network pharmacological analysis. J Pharm Biomed Anal. 2017;141:95-107.

Zhan Y, Li T, Huang W, Jian H, Li Y, He M, et al. Simultaneous determination of caftaric acid, cichorigenin and chicoric acid in Erding Granules by HPLC. Chin Trad Pat Med. 2015;37:2190-3. (Article in Chinese).

Zhu JX, Wang Y, Kong LD, Yang C, Zhang X. Effects of Biota orientalis extract and its flavonoid constituents, quercetin and rutin on serum uric acid levels in oxonate-induced mice and xanthine dehydrogenase and xanthine oxidase activities in mouse liver. J Ethnopharmacol. 2004;93(1):133-40.

Zhu Y. Chinese Materia Medica: Chemistry, Pharmacology and Application. Boca Raton: CRC Press; 1998. p. 207-208.

Zuo J, He H, Zuo Z, Bou-Chacra N, Löbenberg R. Erding Formula in hyperuricaemia treatment: unfolding traditional Chinese herbal compatibility using modern pharmaceutical approaches. J Pharm Pharmacol. 2018;70(1):124-132.

Received for publication on $16^{\text {th }}$ December 2017 Accepted for publication on $28^{\text {th }}$ April 2018 